\title{
Chapter
}

\section{NANODRUG ADMINISTRATION ROUTES}

Letícia Marques Colomé*, Eduardo André Bender, and Sandra Elisa Haas

Federal University of Pampa, Uruguaiana, Brazil 


\section{Contents}

2.1. INTRODUCTION

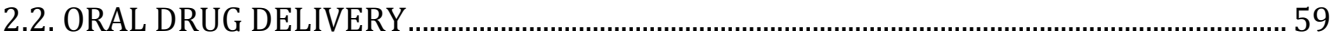

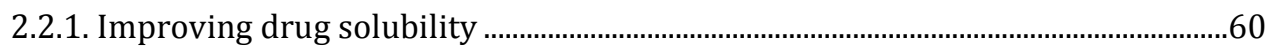

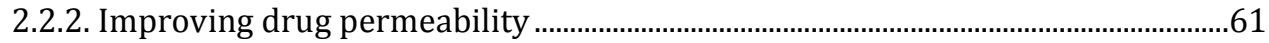

2.2.3. Improving drug stability in the gastrointestinal tract...........................................63

2.2.4. Oral controlled release ............................................................................................64

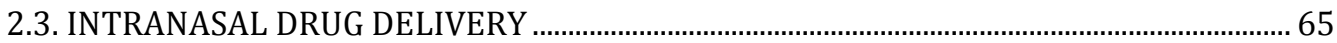

2.3.1. Systemic delivery of peptides and proteins .....................................................................66

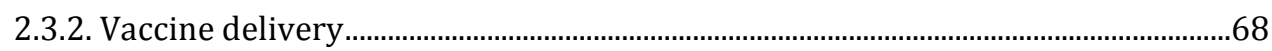

2.3.3. Central nervous system delivery...........................................................................68

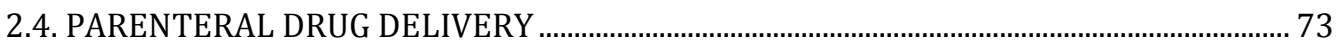

2.4.1. Stealth nanoparticles for the parenteral route ........................................................... 74

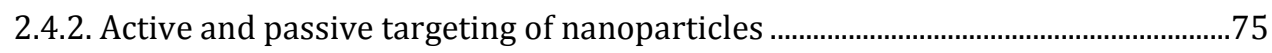

2.5. DERMAL AND TRANSDERMAL DRUG DELIVERY.................................................................. 77

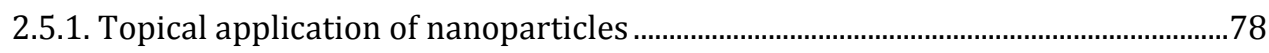

2.5.2. Innovative approaches for cutaneous application of nanoparticles ......................80

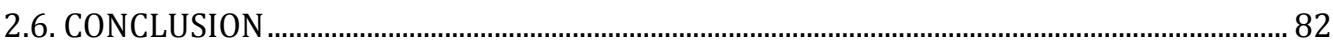

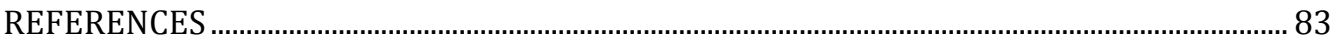




\subsection{INTRODUCTION}

Nanoparticles are colloidal structures less than $1 \mu \mathrm{m}$ in size that have received considerable attention as drug delivery systems. Many drugs and other molecules can be carried in nanoparticles, and these systems can improve pharmacological effectiveness. Obstacles to achieving successful drug therapy are often linked to problems with drug bioavailability. Bioavailability depends on the administration route employed and the absorption and metabolism of the drug. In general, the choice of the delivery route includes aspects such as patient acceptability, the characteristics of the drug, and the accessibility and/or effectiveness of the drug regarding the site of application. Among the different drug delivery routes, the oral route is the most frequently used. However, nasal, ophthalmic, parenteral, dermal, transdermal, pulmonary, and others have also been considered for drug-loaded nanoparticle administration.

According to the administration route used, variations in the pharmacological effects of drug-loaded nanoparticles can occur. Many of these effects are related to physiological and physicochemical situations such as drug transport and metabolism, receptor affinity, membrane permeability, protein binding, gene regulation, and protein expression. The type of colloidal carrier system must also be taken into consideration, since many systems are used for drug transport, e.g. polymeric nanoparticles, liposome vesicles, cyclodextrins, and dendrimers. This review focuses on the different administration routes used for nanosystems in drug therapy, especially considering polymeric nanoparticles.

\subsection{ORAL DRUG DELIVERY}

The oral route is the most widely used pathway for drug delivery. It offers innumerous advantages in comparison to other routes for the patient, such as convenience, painless administration, and self-application, resulting in high compliance. The gastrointestinal tract provides a large surface area for absorption (300-400 $\mathrm{m}^{2}$ ), an excellent blood supply, and extensive residence time, which are advantageous for drug absorption, especially for those with good solubility and permeability [1,2]. However, most drugs have problems of stability, permeability or solubility in the gastrointestinal tract, which results in low bioavailability, erratic absorption, large variations in intra- and inter-subject pharmacokinetics, and a lack of dose proportionality. Therefore, many new therapeutic drugs cannot be developed for use as conventional oral formulations, due to the inhospitable environment found in the gastrointestinal tract, such as high metabolic activity, $\mathrm{pH}$ variations, and the presence of a mucus layer. 
In order to circumvent the limitations associated with the absorption process, attention has shifted to nanotechnology approaches. The encapsulation of drugs (or candidates), peptides, and proteins in polymeric nanoparticles have been used to improve the apparent water solubility, to enhance the intestinal permeability (mainly by the gut uptake), to control drug delivery, and to protect the drugs from gastrointestinal enzymes and local $\mathrm{pH}$.

\subsubsection{Improving drug solubility}

Recent reports estimate that at least $40 \%$ of new drug candidates are poorly soluble in water, resulting in low bioavailability. A great number of delivery systems have been developed to increase the oral bioavailability of these compounds, by increasing the dissolution rate and/or by increasing the dissolved drug levels [3]. Spironolactone is a specific aldosterone antagonist which is used as a potassium sparing diuretic in pediatric patients, but shows incomplete oral absorption because of its low solubility and slow dissolution rate. Therefore, spironolactone-loaded poly( $\varepsilon$-caprolactone) (PCL) nanocapsules were developed to increase the solubility of this drug [4]. Initially, a solubility study of spironolactone was performed in Labrafil ${ }^{\circledR}$, Labrafac $^{\circledR}$ CC, Labrafac ${ }^{\circledR}$ Hydro, Myritol ${ }^{\circledR}$, and olive oil. The solubility was greater in a mixture of $\mathrm{C}_{8} / \mathrm{C}_{10}$ ethoxylated glycerides (Labrafac ${ }^{\circledR}$ Hydro), resulting in a higher encapsulation efficiency, a parameter which is influenced by the solubility of the substance in the oily core of nanocapsules. The percentage of dissolved spironolactone from nanocapsules was $100.51 \%$ in 20 min after dilution of the formulation in simulated gastric fluid. In another study, to improve the intrinsic solubility of the poorly water-soluble drug efavirenz, polymeric micelles based on a mixture of poloxamine (Tetronic T304 and T904) and poloxamer (Pluronic F127) were developed [5]. Solubility factors were calculated by the relation of the apparent solubility of the drug in micelles and its intrinsic solubility in buffer. The highest values for this parameter were obtained with the T904/F127 mixture $(75: 25)$, since these were the most hydrophobic micelles.

The development of nanocapsules is advantageous not only for drugs but also for organochalcogen compounds. Diphenyldiselenide is a selenoorganic compound that is poorly soluble in water, presenting a $\log D$ value of 3.13 [6]. Thus, nanocapsule suspensions containing 1.56 and $5.0 \mathrm{mg} \mathrm{ml}^{-1}$ of the drug were prepared by nanoprecipitation. Using canola oil as the oily core, the encapsulation rate was close to $100 \%$. The intragastric administration of the drug in mice induced concentrations in urine and adipose tissue higher for the diphenyldiselenide nanocapsules than for the free compound, and the opposite was observed in the feces. The results showed that organochalcogen solubilization in nanocapsules improved its bioavailability.

For class II compounds of the Biopharmaceutics Classification System (BCS) (low solubility and high permeability), nanotechnology has been extensively 
applied allowing increased solubility of the compound, reclassifying it as class I (high solubility and high permeability) [7-9]. Celecoxib is a non-steroidal anti-inflammatory drug for pain and inflammation which is classified as a class II compound. Ethylcellulose:casein nanoparticles were prepared by microfluidization and subsequent spray-drying [8]. In vitro non-sink dissolution of celecoxib from nanoparticles in a fasted duodenal model solution showed rapid dissolution, reaching the terminal value within the first minute. On the other hand, bulk celecoxib crystals take at least $1 \mathrm{~h}$ to reach their maximum concentration. In vivo pharmacokinetic testing in dogs and humans showed that time of maximum concentration $\left(T_{\max }\right)$ from nanoparticles and resuspended nanoparticles was twice as fast as the free drug. The bioavailability was $25 \%$ and $75 \%$ for free celecoxib and the nanoparticles, respectively. Since nanoparticles have a higher surface area and a shorter drug diffusion distance for release, high concentrations of the dissolved drug are found at the dissolution site, i.e. the gut.

Felodipine is a drug which exhibits poor oral bioavailability (15\%) due to limited aqueous solubility and extensive first pass metabolism. A $3^{2}$ factorial design was performed to evaluate the influence of felodipine, poly(D,L-lactic acid) (PLA) and Pluronic F-68 on nanoparticle characteristics [9]. The optimized polymer $(1: 20)$ and surfactant concentration $(1.5 \%)$ resulted in nanoparticles presenting the better characteristics in terms of encapsulation efficiency, particle size and zeta potential. Around $60 \%$ and $30 \%$ of the free and nanoencapsulated drug, respectively, were released in intestinal medium, and mathematical modeling suggested a first order release. Intra-gastric administration of the free drug and felodipine nanoparticles in hypertensive rats revealed that the drug delivery system normalized blood pressure and maintained normal levels for up to 3 days. These results could be attributed to enhanced bioavailability due to direct uptake by Peyer's patches in the intestine, as well as the sustained release of felodipine from the polymeric matrix.

\subsubsection{Improving drug permeability}

Chitosan (CS) and its derivatives facilitate nanoparticle uptake and enhance the permeability of drugs via two mechanisms: i) mucoadhesion, by the interaction of their positive surface charge with the anionic components of the glycoprotein on the surface of epithelial cells and ii) tight junction opening, thus increasing paracellular transport $[10,11]$. Thiolated CS coated polymethacrylate nanoparticles were developed using a non-hazardous organic solvent method and two molecular weights of CS (20 and $50 \mathrm{kDa})$. The apparent permeability coefficient was similar for both formulations, and the nanoparticles were 30 -fold better than the free drug regarding their ability to disrupt the membrane of Caco-2 cells [11]. The relative bioavailability of docetaxel-loaded nanoparticles prepared with $20 \mathrm{KDa}$ CS was $68.9 \%$ in comparison to non-encapsulated docetaxel after oral administration in Wistar 
rats. The half-life and area under the plasma concentration time curve from zero (0) hours to infinity $(\infty)\left(\mathrm{AUC}_{0-\infty}\right)$ values for the free and nanoencapsulated drug increased along with permeability and bioavailability for nanoparticulate docetaxel [12].

The mechanism of the absorption and permeability of 7-ethyl-10-hydroxycamptothecin (Sn38)-loaded CS-coated poly(lactic-co-glycolic acid) (PLGA) nanoparticles was investigated. Sn38 is a class IV compound in the BCS, and P-glycoprotein (Pgp) efflux contributes to its low permeability. CS-coated and uncoated nanoparticles were prepared by the oil-in-water emulsion solvent evaporation method and showed a similar particle size, encapsulation efficiency, and drug loading content, but the zeta potential values were influenced by coating with CS. The inhibition of Pgp by verapamil showed that the drug absorption rate was constant and the effective permeability coefficient was similar to the values obtained with CS-coated nanoparticles, suggesting that Sn38 was not recognized by Pgp, but other mechanisms may be involved. An investigation into transcytosis pathways in Caco- 2 cells showed a specific decrease in uptake of 6-coumarin-labeled CS-coated PLGA nanoparticles by the use of sucrose (an endocytosis inhibitor), indicating that these particles use the clathrin-mediated endocytic pathway to escape from Pgp recognition [13].

Aiming to improve the paracellular permeability of hydrophilic compounds, 2-dodecyl-1-yl-succinic anhydride groups were attached to CS to form lauryl succinyl chitosan $\left(\mathrm{C}_{12}-\mathrm{CS}\right)$. Insulin-loaded $\mathrm{C}_{12}$-CS nanoparticles were prepared by ionic gelation. The transepithelial electrical resistance values were reduced in the presence of both the native and derivatized CS nanoparticles. The data were corroborated by confocal laser scanning microscopy images and showed that the interruption of Z01-type tight junctions occurred with both nanoparticle formulations [14].

Curcumin also shows poor solubility and permeability which limits its therapeutic use, despite its numerous pharmacological activities. To counteract these limitations and improve its biological availability, PLGA nanospheres were developed. Curcumin-loaded PLGA nanospheres prepared by modified solid-in-oil-in-water solvent evaporation technique showed an increase in solubility and sustained drug release, especially in intestinal juice, which can be attributed to the fact that curcumin is primarily absorbed in the gut. In the rat gut, the residence time was shorter for nanospheres in comparison to free curcumin, due to the inhibition of Pgp by nanoparticles observed in the in situ single-pass intestinal permeability. After intragastric administration to rats, the AUC of curcumin and curcumin-loaded PLGA nanospheres were 367 and $2066 \mathrm{~min} \mu \mathrm{g} \mathrm{mL}^{-1}$, respectively, resulting in a relative bioavailability of $563 \%$ [15].

One of the pathways for nanoparticle uptake in the gut is through Peyer's patches, which is the gut-associated lymphoid tissue that contains $\mathrm{M}$ cells. 
Through this way, nanoparticles can be delivered to the lymphatic system and then to the circulation. Gemcitabine chlorhydrate loaded PLGA nanoparticles were formulated by a multiple emulsification solvent evaporation method [16]. The permeability of the nanoencapsulated drug was 6.38 times higher than the free drug, evaluated using a Caco- 2 cell monolayer. This increase can be attributed to uptake by Peyer's patches, which was confirmed by confocal microscopy in rat intestinal villi. In agreement, gemcitabine-loaded PLGA nanoparticles showed a 3.24-, 3-, and 21.47-fold increase in maximum drug concentration $\left(C_{\max }\right)$, half-life, and relative bioavailability, respectively, in comparison to the drug solution after oral administration to rats. In another study [17], an in situ intestinal perfusion technique was used to assess the permeability of vancomycin-loaded PLGA nanoparticles. Different drug: polymer ratios were studied, and the permeability was superior for all these formulations in comparison to the free drug.

Active drug targeting is a strategy used to improve the bioavailability of paclitaxel, a BCS class IV compound, by folic acid functionalized PLGA nanoparticles [18]. Paclitaxel transport across Caco-2 cells was significantly increased by the nanoencapsulation (by approximately 8-fold). The intracellular accumulation was also higher for folic acid-PLGA nanoparticles than for free paclitaxel, and it was time dependent, with peak concentrations up to $6 \mathrm{~h}$ after incubation. Another strategy used to improve PLGA nanoparticle permeability is the presence of a stabilizer. Sonaje and co-workers used didodecyldimethylammonium bromide (DMAB) and poly(vinyl alcohol) (PVA) to improve the permeability of ellagic acid [19]. The positive zeta potential, influenced by the presence of DMAB, was substantial enough to improve rat intestinal permeability.

\subsubsection{Improving drug stability in the gastrointestinal tract}

To evaluate whether encapsulation in CS-tripolyphosphate (TPP) nanoparticles enhances the gastrointestinal stability of green tea catechin $(-)$-epigallocatechin gallate, nanoparticles were administered to mice and catechin concentrations were measured in gastric and intestinal samples [20]. The stability of cathechin in nanoparticles were significantly increased by 1.5- and 2.5-fold in the stomach and in the intestinal juice, respectively, when compared with free cathechin. The plasma level was 1.5 times higher for the encapsulated drug than non-encapsulated drug. These results can be attributed to enhanced exposure of cathechin in the jejunum due to the stability increasing effect of CS-TPP nanoparticles.

In the same way, CS nanoparticles prepared by an ionic gelation method using TPP or hydroxypropyl methylcellulose phthalate as the complexing agent demonstrated the advantageous use of a $\mathrm{pH}$-sensitive polymer to improve encapsulated insulin stability [21]. Insulin in solution undergoes total degradation within $5 \mathrm{~min}$ in simulated gastric fluid. In CS-TPP nanoparticles, 
only $10 \%$ of the insulin was protected after $30 \mathrm{~min}$, while $40 \%$ of the drug was protected after $120 \mathrm{~min}$ with CS-hydroxypropyl methylcellulose phthalate nanoparticles. The intestinal mucosal adhesion and uptake of these particles in rats showed higher values in comparison to the free drug. Both insulin-loaded nanoparticle formulations showed significant hypoglycemic effect in rats after oral administration. Insulin-loaded CS-TPP nanoparticles decreased glycemia by 3.5 -fold, whereas for the insulin-loaded CS-hydroxypropyl methylcellulose phthalate nanoparticles, the decrease was 9.8-fold in comparison to the free drug.

Polymeric micelles have been developed to improve the stability, solubility, and bioavailability of docetaxel [22]. The mixed micelles were prepared with poly(ethylene glycol) (PEG)-PLA, D- $\alpha$-tocopheryl, PEG 1000, succinate, and stearic acid grafted with CS oligosaccharide, using a thin film hydration method. After dilution of the micelles in simulated gastric fluid without pepsin $(\mathrm{pH}$ 1.6) and simulated intestinal fluid without trypsin ( $\mathrm{pH}$ 6.5), the particle size showed little modification during $12 \mathrm{~h}$ of incubation. At higher $\mathrm{pH}$, the size was significantly increased after $12 \mathrm{~h}$. The pharmacokinetic parameters after oral administration of free docetaxel and docetaxel incorporated into micelles demonstrated a significant 3 -fold increase in $C_{\max }$ after micelle administration, and the peak was reached in a quarter of the time compared to after administration of the free drug. The relative bioavailability was 2.5 -fold higher.

\subsubsection{Oral controlled release}

Countless polymers can be used in the nanoparticle composition to provide sustained release. Among them, Eudragit ${ }^{\circledR}$ stands out. Eudragit ${ }^{\circledR}$ is the trade name of copolymers derived from esters of acrylic and methacrylic acids, which have numerous applications depending on their functional groups. This versatility makes them extremely used in the pharmaceutical industry. Polymethacrylates are non-biodegradable polymers and are therefore not suitable for parenteral use [23].

Eudragit ${ }^{\circledR}$-based nanosuspensions were developed to control glimepiride release and improve its solubility [24]. Eudragit ${ }^{\circledR}$ RLPO was chosen because it forms positively charged submicron particles. The drug: polymer ratios used (1:5-1:40) influenced glimepiride release evaluated by the dialysis bag diffusion technique using phosphate buffer solution ( $\mathrm{pH}$ 6.8) as medium. All formulations showed a biexponential release profile. In this case, initial release can be attributed to the burst effect, which is due to rapid release of the drug adsorbed on the particle surface, while the second phase is slow and depends on the characteristics of the polymeric matrix. Eudragit ${ }^{\circledR}$ RLPO is $\mathrm{pH}$ independent and undergoes swelling in aqueous media with a consequent increase in permeability and release of the drug. Formulations prepared with a $1: 40$ drug : polymer ratio showed a slower release rate with $95 \%$ and $96 \%$ of the drug released in $24 \mathrm{~h}$, following a Fickian release mechanism. 
Another example of a cationic polymer of the trade name Eudragit ${ }^{\circledR}$ is the Eudragit ${ }^{\circledR}$ RS. Considering its mucoadhesive features, Wu and co-workers [25] developed insulin-loaded PLGA-Eudragit ${ }^{\circledR}$ RS nanoparticles which were used to fill hydroxypropyl methylcellulose phthalate-coated capsules. The shake-flask method was used to evaluate insulin release in two media: simulated gastric fluid ( $\mathrm{pH} 1.2)$ and simulated intestinal fluid ( $\mathrm{pH} 7.4)$. The charge interaction between insulin and Eudragit ${ }^{\circledR}$ RS were responsible for total insulin release in acidic $\mathrm{pH}$ medium and only $50 \%$ in pH 7.4 medium in less than $1 \mathrm{~h}$.

Due to their ability to dissolve only in an environment where the $\mathrm{pH}$ is greater than 7.0 or 6.0, pH-sensitive Eudragit ${ }^{\circledR}$ S100 and Eudragit ${ }^{\circledR}$ L100, respectively, were used to prepare papain-loaded nanoparticles using the water-oil-water emulsion solvent evaporation method [26]. The release rate of papain was $20.71 \%$ (Eudragit ${ }^{\circledR}$ L100) and $13.01 \%$ (Eudragit ${ }^{\circledR} S 100$ ) at pH 6 and $100 \%$ (Eudragit ${ }^{\circledR} \mathrm{L} 100$ ) and $53 \%$ (Eudragit ${ }^{\circledR}$ S100) at pH 7.4, according to Eudragit ${ }^{\circledR}$ solubility. The nanoparticles contributed to the dissolution and diffusion process of papain.

\subsection{INTRANASAL DRUG DELIVERY}

The nasal route has attracted great interest as an alternative route for the administration of diverse agents. Generally, the nasal route is used for the administration of decongestants, antibiotics, and mucolytics. However, the advancement of nanotechnology has enabled studies involving anticancer drugs, analgesics, central nervous system (CNS) drugs, peptides, and diagnostic agents.

The nasal mucosa presents many advantageous characteristics for the systemic absorption of drugs (epithelial microvilli, large surface area, rich vasculature, and a highly porous endothelial membrane) which facilitate drug permeation, such that drugs can be absorbed directly into the systemic circulation without the first pass effect. These characteristics lead to a fast onset of action, quickly reaching therapeutic plasma levels of the drug $[27,28]$. All these factors permit dose reduction, reduce side effects, and increase patient compliance to treatment. Additionally, the intranasal administration provides a non-invasive and painless alternative to the intravenous and oral routes, thus maximizing patient comfort.

Despite the advantages of this pathway, there are some barriers which limit the nasal absorption of drugs that must be considered during the discovery of new chemical entities intended for nasal therapy as well as during the development of nasal formulations. These barriers include mucociliary clearance, which rapidly removes the formulation from the nasal cavity. In addition, enzymatic degradation can occur in both the lumen of the nasal cavity and passing through the epithelial barrier [29]. Other factors that limit drug 
nasal absorption are the low permeability of the epithelium which impedes the transference of polar drugs or high molecular weight substances such as peptides and proteins, the $\mathrm{pH}$ of the system which must be compatible with the nasal cavity, and the small volume that can be administered and the mucus layer.

Nanoparticulate systems, especially polymeric nanoparticles, show many advantages as systems for nasal administration, such as alteration of the mucus layer, alteration of tight junctions, erosion of the mucosal surface, increased drug contact time at the absorption site, the use of bioadhesive materials, and a reduction in the mucociliary clearance rate. [27,30]. The main applications of polymeric nanoparticles for nasal route include the delivery of peptides and proteins, vaccines, and brain targeting.

\subsubsection{Systemic delivery of peptides and proteins}

Peptides and proteins have great therapeutic potential; however, they lack appropriate characteristics for oral administration. Their instability in acid gastric medium, high molecular weight, and hydrophilicity hinder the permeation of peptides and proteins through the gut epithelium. Additionally, the general administration of these macromolecules involves the parenteral route, generating drawbacks such as the requirement for a qualified professional for administration, patient compliance, and high production cost. Thus, the nasal route has emerged as a route of interest to increase the bioavailability of these agents.

Insulin-loaded nanoparticles administered by the nasal route are an alternative to increase the biological half-life and improve the stability and therapeutic efficacy of this protein. Different polymers have been studied for this purpose, especially CS [31]. CS is an attractive material that can confer bioadhesion and increase the absorption of formulations intended for drug delivery in the nasal cavity. Among the methods used to prepare CS nanoparticles, it is highlighted the ionotropic gelation using tripolyphosphate (TPP) ions as the cross linking agent [32-35].

A simple one-step procedure to obtain CS-TPP and concomitant complexion with sodium alginate was developed, aiming to prepare transmucosal formulations for the absorption of insulin. [31]. The pharmacological evaluation in rabbits showed that nasal administration of insulin-loaded CS-TPP nanoparticles induced a rapid decrease in blood glucose, and the presence of alginate in the nanoparticles led to a prolonged hypoglycemic response for up to $5 \mathrm{~h}$. According to the authors, these effects were due to the intracellular delivery of insulin. In addition to mucoadhesion of the nanoparticles due to the characteristics of CS, alginate may have contributed to this mechanism due to its high affinity for $\mathrm{Ca}^{2+}$. Using the same preparation method, insulin-loaded nanoparticles were developed based on a copolymer formed of PEG-CS [33]. Pharmacodynamic/pharmacokinetic experiments in 
rabbits showed that the nanoparticles led to a significant reduction in blood glucose levels that remained at a low concentration for, at most, 2-3 h. In addition, a rapid increase in plasma insulin concentrations occurred compared to that obtained with the copolymer suspension. Another example of hybrid CS nanoparticles are those constituted by CS-sulfobutylether-cyclodextrin [34]. These cationic insulin nanoparticles were able to enter in the nasal mucosa due to their permeation-enhancing properties. The transport of insulin across the nasal barrier led to a significant decrease in the plasma glucose levels.

A strategy to improve the mucoadhesive and permeation properties of unmodified CS is the covalent attachment of thiol-bearing groups. Krauland and co-workers [36] developed insulin-loaded CS-4-thiobutylamidine nanoparticles and showed that after nasal administration in non-diabetic rats, the plasma concentrations of insulin were higher than with unmodified CS-nanoparticles. Insulin-loaded CS-4-thiobutylamidine nanoparticles led to a more than 1.5-fold higher bioavailability and more than 7-fold higher efficacy in decreasing glycemia. In another study [37], insulin-loaded trimethyl-CS nanocomplexes were prepared. The ratio between insulin and the derivated polymer in the formulation influenced both the bioavailability and nasal epithelial integrity. The best absorption values were obtained using the proportion $1: 30.6$ (insulin:trimethyl-CS), but this formulation also led to severe damage to the nasal cavity in rats. In comparison, PEGylated trimethyl-CS copolymers were used to prepare insulin nanoparticles with similar efficacy but exhibited a mild level of epithelial damage with slight mucus secretion and goblet cell distension.

Nanoparticles based on polymers having characteristics of mucoadhesion and enzyme inhibition have been used to encapsulate insulin. Phenylboronic acid-functionalized glycopolymers exhibit potent inhibition activities against serine proteases such as trypsin, chymotrypsin, elastase, and leucine aminopeptidase and have been used to synthesize poly(3-acrylamidophenylboronic acid-ran- $N$-maleated glucosamine) nanoparticles [38]. These nanoparticles adsorbed high amounts of mucin, which was attributed to the interaction between the phenylboronic acid groups and sialic acid residues of the mucin. All formulations induced a decrease in blood glucose levels $9 \mathrm{~h}$ after nasal administration in rats. Images of the interaction of fluorescein isothiocyanate (FITC)-insulin-loaded nanoparticles with the rat nasal epithelium by confocal laser scanning microscopy indicated that endocytosis plays an important role in insulin transmucosal delivery. CS- $N$-acetyl-L-cysteine nanoparticles were prepared by in situ ionic gelation of CS with TPP [32]. The mucoadhesion of the nanoparticles prepared with $N$-acetyl-L-cysteine was around 2-fold higher than unmodified CS nanoparticles. In addition, the total decrease in plasma glucose levels was $16.2 \%$ within $5 \mathrm{~h}$, which was significantly higher than the value obtained with unmodified insulin nanoparticles (8.3\%) [39]. 


\subsubsection{Vaccine delivery}

Current immunization methods involve parenteral and intramuscular administration of antigen. Mucosal immunization has several advantages in comparison to these routes, such as needle-free administration and the possibility of self-administration. These features eliminate the necessity for trained personnel for vaccine administration and improve patient compliance in comparison to the parenteral route. The nasoepithelium has low enzymatic activity, $\mathrm{pH}$ values close to neutral, moderate permeability, and high availability of immune-reactive sites [40]. However, the viability of free antigens is low because of permeability problems and a reduced ability to stimulate the innate and adaptive immune system. Thus, the use of adjutants, such as polymeric nanoparticles, is an alternative to bypass these disadvantages, thanks to improved residence time and contact with the mucosa, delivering the antigens directly to lymphoid tissues. Table 1 shows recent advances in the nasal delivery of vaccines based on nanoparticles.

\subsubsection{Central nervous system delivery}

The blood-brain-barrier (BBB) is located at the interface between the brain and the vessels of the circulation and is the most important structure connecting the central nervous systems with peripheral tissues. The main characteristic of this barrier is the existence of an endothelium with very restricted permeability as well as the presence of enzymes in large amounts. Only water, gases such as oxygen and carbon dioxide, and certain fat-soluble and very small hydrophilic molecules pass from the blood to the brain [41]. Therefore, the BBB functions as an interface that limits and regulates the exchange of substances between the blood and the central nervous system for $100 \%$ of large molecule neurotherapeutics and more than $98 \%$ of all small molecule drugs [42], making it difficult to reach therapeutic concentrations of drugs in the brain via the general circulation. Thus, the nasal route is an alternative to circumvent these limitations.

Designated nose-to-brain transport, the local key is the olfactory region, which is located at the top of the nasal cavity and is extremely porous, allowing the passage of neuronal bundles from the nasal region to the brain [43]. These nerves connect the nasal passages to the brain and spinal cord and, as well as the vasculature, cerebrospinal fluid, and lymphatic system, contribute to transportation of molecules to the CNS following adsorption from the nasal mucosa. Besides the olfactory nerve, the trigeminal nerve plays a fundamental role, facilitating the translocation of drugs via sensory fibers. The trigeminal nerve is formed by the ophthalmic branch that innerves the anterior and upper parts of the nasal cavity, the maxillary branch located on the respiratory nasal mucosa, and the parasympathetic fibers that accompany the sensory nerves to the sphenopalatine ganglion $[29,44]$. 
Despite the advantages of this pathway, transmucosal drug delivery is still limited by the physiological characteristics of nasal administration, such as mucociliary clearance and low permeability. Aiming to increase the availability of the drug by modification of the residence time and permeability, the use of bioadhesive agents and absorption enhancers in nanosized formulations for brain uptake have been used. In addition, nanoparticles are able to protect the encapsulated drug from biological and/or chemical degradation, and extra cellular transport by P-glycoprotein efflux. Their small size potentially allows nanoparticles to be transported by the transcellular pathway through olfactory neurons to the brain or via the various endocytic pathways of sustentacular or neuronal cells in the olfactory membrane. Thus, studies involving nanoparticles for nose-to-brain drug transport have increased over the last decade and represent a promising strategy for use in diseases such as schizophrenia, depression, epilepsy, meningitis, migraine, neuro-AIDS, brain cancer, and neurodegenerative diseases.

Taking account the advantages of the CS polysaccharide such as mucoadhesion and enhanced permeation, countless articles have studied the application of this atoxic polymer in CS nanoparticles for nose-to-brain transport. Thymoquine-loaded CS nanoparticles were developed to avoid first-pass metabolism and improve its distribution to the brain with sustained action [45]. CS nanoparticles were prepared using the ionic gelation process. The optimized formulation containing 1.5:1.5:2 thymoquine:CS:TPP was evaluated in an ex vivo permeation assay using goat nasal mucosa; it was found that the maximum permeation was 3 -fold higher for nanoencapsulated thymoquine in comparison to free drug. In the same direction, the relative bioavailability (nose-to-brain) evaluated in rats showed a 12 -fold increase in comparison to thymoquine intranasal solution.

Rivastigmine is a hydrophilic drug used in Alzheimer's disease because it is an inhibitor of acetylcholinesterase enzyme. Rivastigmine-loaded CS nanoparticles were prepared by the same method cited above [46]. A biodistribution study in rats found that the brain-blood ratios $30 \mathrm{~min}$ after administration were 0.790 and 1.712 for the rivastigmine intranasal solution and the drug-loaded nanoparticles, respectively, indicating direct nose to brain transport bypassing the BBB. Comparing to brain concentrations of nanoencapsulated rivastigmine after intravenous and intranasal administration, the results showed that the concentrations were higher for the extravascular in comparison to the intravascular route, highlighting the advantage of nose-to-brain transport. In another study, the plasma and cerebrospinal fluids of free estradiol and estradiol encapsulated in CS nanoparticles were investigated after intravenous and nasal administration in rats [32]. The concentration of estradiol in the cerebrospinal fluid after intranasal administration was higher than after intravenous administration. Besides, the time to reach this concentration was very fast by the nasal route 
using nanoparticles, showing that CS allows for a rapid onset of action in the CNS.

The apparent permeability coefficient of tizanidine solution in a human nasal septum carcinoma cell line (RPMI 2650 cells) was strongly increased when the drug was encapsulated in thiolated CS nanoparticles [41]. The brain: blood ratio of tizanidine-loaded thiolated CS nanoparticles was 1.92 after $30 \mathrm{~min}$ and $C_{\max }$ was significantly higher than that obtained with the free drug. These results emphasized the importance of the mucoadhesion effect of thiolated CS, which increased the mean residence time in the nasal cavity. Another reason for the improvement in brain drug uptake may be the inhibition of CYP450 activity present in the nose, due to the ability of the thiol group to inhibit the metabolism of this enzymatic group. In another study, the permeability of free and leucina-enkephalin loaded $N$-trimethyl CS nanoparticles were evaluated in the porcine nasal mucosa, showing a 35 -fold increase in comparison to the free peptide [47]. Furthermore, leucina-enkephalin was labeled with the fluorophore 4-fluoro-7-nitrobenzofurazan and instilled into the nostrils of mice to investigate brain penetration. The results showed that, after $60 \mathrm{~min}$, significant fluorescence was visualized in brain sections. These observations were corroborated by antinociceptive tests that demonstrated a superior response with the use of nanoparticles. The authors suggested that the positive charge of $\mathrm{N}$-trimethyl CS on the nanoparticle surface induced an electrostatic interaction with the anionic binding sites of the brain capillaries and transferred the labeled peptide into the brain. Moreover, CS formed a hydrophilic corona around the nanoparticles, preventing uptake by the mononuclear phagocytic system and increased the residence time of the drug in the body.

PEG surface modification is associated with improving nose-to-brain delivery of encapsulated agents in pegylated nanoparticles [48-52]. Likely, the PEG chains easily penetrate the mucus layer, thus preventing the degradation of particles and allowing access to epithelial cells in the olfactory region [43]. Other factors can influence the ability of PEG-coated nanoparticles to undergo nose-to-brain transport, such as the anchorage of ligands. Lactoferrin, a natural iron binding cationic glycoprotein of the transferrin family, is expressed in various tissues and also in the brain cells, such as brain endothelial cells and neurons. Using an emulsion-solvent evaporation technique, Liu and co-workers [52] developed lactoferrin conjugated PEG-co-PCL nanoparticles and encapsulated 6-coumarin as a model drug. PEG-Co-PCL nanoparticles showed increased time-dependent uptake compared to naked nanoparticles within $6 \mathrm{~h}$ in the human bronchial epithelial cell line (16HBE14o-cells). Small interfering RNAs (siRNAs) have been extensively researched to treat CNS diseases, but the stability and cell penetration ability of these molecules are limited. To this purpose, another study [53] developed PEG-co-PCL nanomicelles conjugated with a cell-penetrating peptide named Tat-G. Concentrations in the cerebrospinal fluid were significantly higher for 
conjugated nanomicelles in comparison to naked nanomicelles. In agreement, after nasal administration in rats, the nose-to-brain pathway involving the olfactory and trigeminal nerves showed the ability of these systems to permeate the nasal mucosa and to facilitate the brain delivery of nucleic acids.

Lectin surface modification is a way to improve brain delivery, since lectins are proteins or glycoproteins that have selective affinity for biological surfaces. One example of lectin is the wheat germ agglutinin (WGA) which presents the ability to specifically bind to $N$-acetyl-D-glucosamine and sialic acid present in the nasal cavity. WGA-PEG-co-PLA nanoparticles were prepared at a $1: 3$ molar ratio of WGA : maleimide by an emulsion-solvent evaporation technique. The ciliotoxicity was evaluated in vivo in a rat nasal mucosa model and it was comparable to the negative control [49]. The immunogenicity and toxicity induced by WGA nanoparticles were evaluated in vivo in the rat nasal cavity. WGA and naked nanoparticles induced a significant increase in brain glutamate levels, but only conjugated nanoparticles exhibited lactate dehydrogenase activity in the olfactory bulb, indicating possible neurotoxicity. The levels of interleukin 8 (IL-8), tumor necrosis factor alpha (TNF- $\alpha$ ), immunoglobulin G (IgG) and immunoglobulin A (IgA) in the rat olfactory bulb and brain remained similar to control and significantly lower than WGA alone [50]. However, the molar ratio of WGA : maleimide played an important role in these results, suggesting that an increase to $1: 10$ would be sufficient to provide the most efficient uptake and mild cytotoxicity [54]. The uptake occurred along olfactory nerves and trigeminal nerves within $2 \mathrm{~h}$ following intranasal administration and the cerebrospinal fluid pathway was not important in this delivery method [51]. 
Table 1. Recent studies for immunization via nasal route

\begin{tabular}{|c|c|c|}
\hline Reference & Polymer & Antigen \\
\hline$[55]$ & $\mathrm{CS}$ & Anti-caries DNA \\
\hline$[56]$ & $\mathrm{CS}$ & $\begin{array}{l}\text { DNA Mycobacterium } \\
\text { tuberculosis }\end{array}$ \\
\hline [57] & $\mathrm{CS}$ & Plasmid pVAXN \\
\hline$[58]$ & $\mathrm{CS}$ & Pneumococcal surface antigen $\mathrm{A}$ \\
\hline [59] & $\mathrm{CS}$ & Dermatophagoides farinae \\
\hline$[60]$ & $\begin{array}{c}\text { CS } \\
\text { Mannosylated-CS }\end{array}$ & $\begin{array}{l}\text { Plasmide DNA anti-gastrin } \\
\text { releasing peptide }\end{array}$ \\
\hline [61] & CS-PCL & H1N1hemagglutinin protein \\
\hline$[62]$ & $\begin{array}{c}\text { CS } \\
\text { Trimethylated chitosan }\end{array}$ & Hepatitis B surface antigen \\
\hline$[63]$ & $\begin{array}{c}\text { Reacetylated } N \text {-trimethyl CS } \\
N \text {-trimethyl CS }\end{array}$ & Ovalbumin \\
\hline [64] & $\begin{array}{c}\text { Trimethyl CS/ hyaluronic acid } \\
\text { Thiolated trimethyl CS/ } \\
\text { Thiolated hyaluronic acid }\end{array}$ & Ovalbumin \\
\hline [65] & $\begin{array}{c}\text { CS } \\
\text { Trimethyl CS } \\
\text { Tri-methylated CS }\end{array}$ & Hepatitis B surface antigen \\
\hline$[66]$ & Trimethyl CS & CpG DNA \\
\hline [67] & Ovalbumin-Trimethyl CS & Ovalbumin \\
\hline [68] & $\begin{array}{l}N \text {-trimethyl-chitosan-mono- } N \text { - } \\
\text {-carboxymethyl chitosan }\end{array}$ & Tetanus toxoid \\
\hline [69] & $\begin{array}{c}\text { PLGA } \\
\text { CS-PLGA } \\
\text { Glycol-CS-PLGA }\end{array}$ & Hepatitis B surface antigen \\
\hline$[70]$ & Poly(anhydride) & Shigella flexneri \\
\hline$[71]$ & Mannosylated Poly(anhydride) & Brucella ovis antigen \\
\hline$[72]$ & $\begin{array}{c}\text { Poly(methylvinylether-co-maleic } \\
\text { anhydride) } \\
\text { Poly(anhydride) }\end{array}$ & Brucella ovis antigen \\
\hline
\end{tabular}

CS: chitosan; TPP: tripolyphosphate; PCL: poly( $\varepsilon$-caprolactone);

PLGA: poly(lactic-co-glycolide acid) 


\subsection{PARENTERAL DRUG DELIVERY}

Generally, the therapeutic results achieved with orally administered drugs require the application of higher doses than those that would be required to achieve the same effect using the parenteral route. This is mainly because the parenteral pathway allows for the rapid and complete absorption of drugs, unlike what happens in the oral route where a large fraction of the dose is lost due to first-pass liver metabolism. Furthermore, the parenteral route allows the use of lower doses and has extended therapeutic effects in comparison to other routes [73,74]. Thus, it is also expected to lower the incidence of side effects compared to oral administration [74].

Drugs with poor dissolution properties are generally promising candidates to have their effect expanded by parenteral administration. These molecules are often difficult to formulate using conventional approaches and are associated with formulation-related performance issues, e.g. poor bioavailability, lack of dose proportionality, slow onset of action, and other attributes leading to poor patient compliance $[73,75]$. Other advantages of parenteral administration are the continuous infusion of drugs with a short half-life as well as drug administration to unconscious and comatose patients. [73,74].

Considering the features of the parenteral route, several studies using nanosystems have been conducted aiming to improve the therapeutic performance of the drugs and/or counteract the issues related to chemical or physical disadvantageous characteristic of the compounds. Countless molecules can be administered in nanoparticles. Cardiovascular agents, anticancer, anti-inflammatory, antibiotic, immunomodulatory, and immunostimulatory drugs, antiglaucoma compounds, and even peptides have been encapsulated in nanoparticles proposed for parenteral route. As a result, their pharmacological effects have been improved or their side effects have been reduced in comparison to the free drugs [75-78].

In one study, nanocapsules were labelled with technetium-99m (99m Tc) and rhenium-188 (188Re) and evaluated in terms of biodistribution parameters after intravenous injection in rats. The results obtained by dynamic scintigraphy showed predominant hepatic uptake, and an ex vivo evaluation indicated a long circulation time of labelled nanocapsules [79]. In another study conducted by Danhier and co-workers [80], it was hypothesized that nanosuspensions could be promising for the delivery of the poorly water soluble anti-cancer multi-targeted kinase inhibitor MTKi-327. The nanosuspension was administered by the parenteral and oral routes and it was observed that the highest regrowth delay of A-431-tumor-bearing nude mice occurred when the nanosuspension was administered intravenously. In this study, it was clear that nanoparticles can be used to increase drug efficacy.

Nanoparticles administered by the parenteral route can be quickly captured by the mononuclear phagocyte system (MPS). In this case, blood cells such as monocytes, leukocytes, and platelets, as well as resident phagocytes such as 
the Kupffer cells of the liver and spleen macrophages, play an important role [81-83]. Thus, organs such as the spleen and liver prevent unrestrained circulation of nanoparticles in the blood, which could otherwise reach several sites in the body $[83,84]$.

\subsubsection{Stealth nanoparticles for the parenteral route}

An approach to distributing nanoparticles to different sites of the body is the use of furtive (to the immune system) nanostructures. Thus, the rapid adhesion of plasma proteins and the consequent capture of nanoparticles by the MPS can be avoided by reducing the particle size, as well as using compounds with hydrophilic surface characteristics. In this way, the introduction of long hydrophilic polymer chains and non-ionic surfactants at the nanocapsule surface can lead to slow opsonization due to a steric effect, delaying essential electrostatic and hydrophobic interactions to bind opsonins onto the nanoparticle surface $[85,86]$. As a consequence, the half-life circulation of the nanoparticle in blood increases [84]. Steric shielding can be obtained using polymers such as polysaccharides, polyacrylamides, PVA, poly( $N$-vinyl-2-pyrrolidone), PEG, PEG-block copolymers, and PEG-containing surfactants (e.g. poloxamines, poloxamers and polysorbates). PEG is the most effective and commonly used strategy to obtain stealth nanoparticles, reducing or delaying the time of recognition and capture of nanoparticles by the MPS $[80,84,86,87]$. Nanoparticles with stealth ability allow for a reduction in drug dose, and consequently, decrease the adverse effects due to better delivery of the drug to the site of action $[83,87,88]$.

A large amount of work has aimed at increasing the half-life of nanocarriers in the bloodstream, and changing their biodistribution, allowing them to reach other cells and tissues such as solid tumors and sites of inflammation [77,83,88-90]. One study prepared a nanoparticle formulation (nanoerythrosomes) containing the antimalarial drug pyrimethamine for intravenous application [81]. The biodegradable, long circulating carrier allowed for controlled and stable drug release, improving the treatment of malaria. In another study, the authors evaluated the antitumor effect, biodistribution profile, and tumor penetration of docetaxel-loaded PEG-PCL nanoparticles using a hepatic cancer model. The prepared nanoparticles were effectively transported into tumor cells by endocytosis and they accumulated around the nuclei in the cytoplasm. In addition, the in vivo biodistribution evaluation performed on tumor-bearing mice by real-time near infrared fluorescence (NIRF) imaging showed that the nanoparticles reached higher concentrations and were retained longer in the tumor than in non-targeted organs after intravenous injection [91].

The ability of nanoparticles to circulate in the bloodstream for a prolonged period of time is a prerequisite for successful therapy [85]. In this context, paclitaxel-loaded PLGA-CS-PEG nanoparticles have been investigated [87]. The 
proposed nanosystem was able to encapsulate a hydrophobic drug and was taken up by phagocytosis. Thus, there was a reduction in opsonization by blood proteins, increasing the bioavailability of the drug. The results suggest that the PEG-CS coating may be a significant step in the development of long-circulating drug carriers for drug delivery into tumors.

A study conducted by Mosqueira and co-workers [92] evaluated the pharmacokinetics and efficacy of intravenously administered halofantrine-loaded nanocapsules prepared from a PLA homopolymer or PLA-PEG. The results showed that while the parasitemia decreased rapidly with the PLA nanocapsules, the effect was more sustained with PLA-PEG nanocapsules. In addition, nanocapsule administration resulted in a suitable halofantrine profile in the plasma, reduced the intravenous dose necessary for the therapeutic effect and, consequently, reduced the toxicity. Thus, these results demonstrate that the application of halofantrine-loaded nanoparticles by the parenteral route can be useful in severe malaria. In this case, is evident that nanoparticles were able to decrease uptake by the MPS because of steric stabilization afforded by PEG linked to the nanoparticle surface $[83,92,93]$.

\subsubsection{Active and passive targeting of nanoparticles}

Different studies have evaluated the ability of nanoparticles to target several sites in the body after parenteral administration. Targeting ability is a major breakthrough in therapy because it is associated with many advantages such as reduced side effects. In passive targeting, the nanoparticles move freely through the vascular system and may randomly pass through the pores of the endothelium, especially in pathological situations. The plasma circulation half-life should be enough for them to reach the tissue passively. Colloidal systems administered intravenously may have a residence time in the bloodstream controlled by chemical changes on the surface of the particulate system $[80,93]$. Particles with furtive characteristics, such as those coated with PEG or biocompatible substances (e.g. peptides and lipids), present advantageous characteristics in this case $[73,74,80,87,89]$.

Likewise, there is the possibility of surface functionalization in nanoparticles with substances such as antibodies, antibody fragments, carbohydrates, peptides, glycolipids, folic acid, mannitol, and genetic material. In the active targeting, the nanoparticle is selectively recognized by receptors on the surface of cells. Since ligand-receptor interactions can be highly selective, which would allow for a more precise targeting of a specific site in the body [76,77,90,93-96]. The approaches using active vectorization with nanoparticles are a quite challenging, and are currently one of the main goals of several studies in nanotechnology. The main findings in the literature show that surface functionalization with nanoparticles offers maximum therapeutic activity, prevents the degradation or inactivation of drugs while on route to the active site, and avoids several other inappropriate reactions $[90,95,97]$. Since 
these kinds of nanoparticles have a specific binding site, they are able to prevent premature binding by plasma proteins and the consequent phagocytic capture more easily than other furtive nanosystems. Additionally, these nanoparticles present the potential to reform the drug development landscape since they can improve drug solubility, change undesirable pharmacokinetics, and increase drug accumulation in target organs and tissues [91,97,98].

The major focus of studies with active vectorization involves therapies and diagnosis for cancer. Considering the complexity of the cancer microenvironment and cancer immune responses, several anti-tumor strategies can be conjugated in order to induce a stronger and more complete anti-tumor immune response [80]. To improve the biodistribution of cancer drugs, nanoparticles have been designed with optimal size and surface characteristics to increase their circulation half-life in the bloodstream $[89,91]$. In addition, nanoparticles directed specifically to a site in the body can avoid systemic toxic effects and significantly enhance the maximum dose tolerated by patients [99].

Among the targeted ligands for cancer therapy, monoclonal antibodies are one of the most commonly used on the nanoparticle surface due to their high specificity and affinity for target antigens [75,100]. The efficiency of chemotherapeutic drugs or toxins targeted to the tumor is based on the binding and internalization of these conjugates into the target cell [75]. Torrecilla and co-workers [78] developed PEG-CS nanocapsules conjugated to the monoclonal antibody anti-TMEFF-2 for targeted delivery of docetaxel. In this study, free docetaxel exhibited a fast and short effect on tumor volume reduction, while bioconjugate nanocapsules with the monoclonal antibody showed delayed and prolonged action with no significant side effects.

Arias and co-workers [93] developed pentamidine-loaded nanoparticles based on PEG covalently attached to PLGA. This complex was coupled to a single domain heavy chain antibody fragment (nanobody) that specifically recognizes the surface of the protozoan pathogen Trypanosoma brucei. In the in vitro effectiveness assay, the results showed that the $50 \%$ inhibitory concentration $\left(\mathrm{IC}_{50}\right)$ was decreased by 7 -fold for the nanobody in comparison to the free drug. Furthermore, an in vivo evaluation using a murine model of African trypanosomiasis showed that the formulation healed all infected mice at a 10 -fold lower dose than the minimal full curative dose of free pentamidine; for $60 \%$ of the mice, this occurred at a 100-fold lower dose. These results show that an active vectoring system based on nanoparticles applied parenterally has the ability to improve conventional therapy.

In another study [94], a ligand metal-CS-lecithin complex was prepared as a new strategy to functionalize the surface of PCL nanoparticles. The results showed that the nanoparticulate complex was able to connect recombinant antibody fragments, known as anti-electronegative LDL single-chain fragment 
variable $[\mathrm{scFv}$ anti-LDL(-)]. This complex was able to react with $\operatorname{LDL}(-)$ cholesterol molecules, making it an important therapeutic tool.

There are many advantages reported in studies evaluating the application of nanoparticles by the parenteral route in therapeutics and diagnosis $[79,89,93]$. However, basic assessments of compatibility of these nanoparticulate systems with the constituents present in the blood circulation are still somewhat deficient. Thus, nanoparticles that aim toward parenteral application should be evaluated with respect to biocompatibility. Polymeric nanoparticles employing biocompatible materials, when administered by the parenteral route, decrease the probability of incompatibilities $[80,88]$. But in any case, the concentration of the material, the presence of other incompatible materials, or unexpected reactions with the constituents of the formulation may make it unstable [82].

One study evaluated the hemocompatibility of the formulations of polymeric lipid-core nanocapsules stabilized with polysorbate 80-lecithin and uncoated or coated with CS. In vitro hemocompatibility studies were carried out with mixtures of nanocapsule suspensions in human blood at $2 \%$ and $10 \%(\mathrm{v} / \mathrm{v})$. The results showed that the ability of plasma samples to activate the coagulation system was maintained in the presence of the lipid-core nanocapsule. The hemolysis values remained restricted to the recommended limits (1\%) when whole blood was incubated with either uncoated or coated CS-nanoparticles at $2 \%$. On the other hand, when the nanoparticles were added to blood at $10 \%$, hemoglobin was readily released into the extracellular environment. According to the authors, this result could be explained by a shift in polysorbate 80 from the colloids to cells or by the interaction of CS with cells due to the high concentration used (10\%), causing hemolysis [82].

Therefore, several molecular reactions may occur that destabilize nanoparticulate systems in a biological medium. The parenteral route is an excellent alternative for nanoparticle administration, but aspects of the chemical nature of the compounds used alone or in combination should always be taken into account.

\subsection{DERMAL AND TRANSDERMAL DRUG DELIVERY}

Nanoparticle application has been primarily focused on parenteral and oral applications. Nowadays, besides these uses, nanosystems applied to the skin are attracting more and more attention from researchers, considering the advantages of nanoparticles for dermal application such as the protection of incorporated active compounds against chemical degradation and flexibility in modulating the release of the compound [101]. Nanoparticles applied to the skin can have one of two desired effects: local activity within the skin (dermal drug delivery) or systemic activity after nanoparticle permeation through the skin (transdermal drug delivery) [102]. 
In both the dermal and transdermal routes, the stratum corneum is the main barrier of the skin that has to be overcome for suitable drug delivery [102]. The skin is composed of the epidermis, dermis, and subcutaneous tissue. The epidermis is again subdivided into four layers (the strata corneum, granulosum, germinativum, and basale). The cells in the stratum basale divide continuously to produce new keratinocytes that move to the outer layers and form the stratum corneum, which is a horny layer of dead cells. In addition to keratinocytes, the viable epidermis contains cells with roles such as melanin production (melanocytes), sensory perception (Merkel cells), immunological function (Langerhans) and the appendages. The appendages include the pilosebaceous units such as hair follicles and associated sebaceous glands, apocrine and eccrine sweat glands [103].

The main role of the skin is to exert defensive mechanisms (physical, immunological, metabolic, and UV-protective barriers) counteracting attacks by microbes, toxic chemicals, UV radiation, and particulate matter [103]. However, the large surface area and easy accessibility of the skin make it an attractive route for drug delivery. Three main routes in the skin have been identified for the penetration of substances. These penetration pathways into and through the skin are separated to the intracellular (across the corneocytes), intercellular (by the lipid bilayers that surround the corneocytes), and transappendicular (that includes hair follicles and sweating gland) routes.

The intercellular route is recognized as the most feasible pathway. However, substances with a molecular weight greater than $500 \mathrm{Da}$ and ionic substances accumulate in appendicular organs, since these substances have great difficulty passing through the stratum corneum [104]. In fact, dominance amongst the three pathways depends on the drug (its solubility, diffuseability, molecular size and physico-chemical properties) and the system used for delivery of the substance [105]. Considering this statement, many researchers have made efforts to develop drug delivery systems for topical application able to target drug molecules to specific skin layers or to the systemic circulation.

\subsubsection{Topical application of nanoparticles}

Despite the benefits of transdermal drug delivery systems, including simple administration, avoidance of uncomfortable intravenous administration, escape from the first pass effect in the liver, and controlling the dose of drug delivery, the development of a transdermal delivery formulation has to consider two critical issues: the physical barrier of the stratum corneum and the hydrophilicity and numerous types of enzymes present in the chemical barrier of the epidermis [104]. Despite the efficiency of therapeutic agents using the transdermal route for both systemic delivery and local delivery, many techniques have been used to enhance the permeability of drug molecules, including nanoparticles. 
Marimuthu and co-workers [106] developed PLGA nanoparticles intended for the transdermal application of encapsulated glucosamine, which is a highly hydrophilic and poor permeable drug. The nanoparticles were prepared by self-assembly of PLGA-glucosamine which was facilitated by probe sonication followed by reversible locking. The authors hypothesized that the nanoparticle's flexibility was due to its structure (hydrophobic PLGA assembly on the outer surface and hydrophilic glucosamine in the inner core). This flexibility helps the nanoparticles to permeate through the skin lipid membrane and release the drug in a sustained manner. In comparison to glucosamine solution, nanoparticles exhibited a better permeation profile and demonstrated a shorter lag time with a higher flux value in ex vivo transdermal permeation. In another work, polymeric nanoparticles were prepared using CS, PLA, and PCL by a solvent extraction method in an attempt to provide prolonged delivery of repaglinide, a hypoglycemic drug [107]. The optimized PLA-repaglinide nanoparticles loaded in transdermal patches induced a reduction in plasma glucose levels and were 76 -fold more effective than conventional oral administration in diabetic rats.

In addition to transdermal patches, semi-solid vehicles make a suitable formulation for nanoparticles administration to the skin. Contri and co-workers [108] evaluated the effect of the encapsulation of capsaicinoids (capsaicin and dihydrocapsaicin) in nanocapsules, as well as the effect of the incorporation of capsaicinoid-loaded nanoparticles in a CS hydrogel, on skin adhesion and skin penetration/permeation. The in vitro skin adhesion experiments showed lower washability for the CS hydrogel containing capsaicinoid-loaded nanocapsules in comparison to the CS hydrogel containing the free drug and hydroxyethyl cellulose containing drug-loaded nanocapsules. The adhesion assay results predicted the skin penetration/permeation behavior, since the CS gel containing nanocapsules led to a higher amount of capsaicinoids in the epidermis and dermis. In a similar work, PLGA nanoparticles and lecithin/CS nanoparticles containing betamethasone-17-valerate enhanced the amount of the drug in the epidermis when compared with the commercial formulation [109]. When nanoparticles were diluted in CS gel, accumulation in skin layers from both gel formulations was higher than the commercial formulation. In addition, both formulations significantly improved anti-inflammatory and skin-blanching effects in comparison to the commercial cream. In another study, a Pluronic F127 hydrogel containing lidocaine-loaded PCL-PEG-PCL nanoparticles was prepared, aiming transdermal application [110]. The efficiency of the local anesthetic, evaluated by the tail-flick latency test in rats, was better for the nanoparticle based hydrogel in comparison to the conventional treatment (cream) with or without focal ultrasound pretreatment.

In some cases, it is necessary to increase the ratio of the drug in the target tissue relative to systemic exposure to ensure successful drug targeting. It is especially important for drugs that require chronic use or drugs that produce 
significant side effects at other body sites [111]. In this context, CS nanoparticles containing hydrocortisone were administered percutaneously to improve transcutaneous absorption of the drug [112]. The hydrocortisone-loaded nanoparticles reduced the corresponding flux and permeation coefficient of the drug across mouse skin in ex vivo experiments, while they exhibited a higher epidermal and dermal accumulation of the drug in comparison to control groups.

In another study, Lee and co-workers [113] developed a core-shell nanoparticle (PLGA core and a positively-charged glycol CS shell) for use as a DNA carrier intended for transdermal delivery into the epidermis via gene gun. The in vivo evaluation using a mouse model demonstrated that bombardment of nanoparticles transfected DNA directly into Langerhans cells present in the epidermis, which migrated and expressed the encoded gene products in the skin draining lymph nodes. It is emphasized that Langerhans cell migration was detected by fluorescent quantum dots loaded into the core of the nanoparticle. Therefore, nanoparticles have the potential for use in immunotherapy and vaccine development and can be an important approach for monitoring functional aspects of the immune system.

\subsubsection{Innovative approaches for cutaneous application of nanoparticles}

Nanoparticle-based topical delivery systems have been demonstrated to be successful approach for topical (into the skin strata) and transdermal (to subcutaneous tissues or into the systemic circulation) delivery. Beside this, the targeted delivery of encapsulated drugs to hair follicle stem cells, such as iontophoresis and microneedle array technologies, has been employed [114].

Indomethacin-loaded PLGA nanoparticles were prepared with or without (bare nanoparticles) a PVA covering by an antisolvent diffusion method with preferential solvating or an emulsification-solvent evaporation method, respectively [104]. The authors evaluated the effectiveness of the nanoparticles for ex vivo iontophoretic transdermal drug delivery. Both nanoparticles presented an average diameter of $100 \mathrm{~nm}$. Bare nanoparticles did not have a hydrophilic stabilizer on the surface, presenting high hydrophobicity and negative charges. The cumulative indomethacin amounts that permeated through rat skin were significantly increased by using either kind of nanoparticles when iontophoresis was applied. However, the bare nanoparticles presented significantly higher permeability in comparison to the PVA-coated nanoparticles, showing that the combination of a bare nanoparticle system with iontophoresis was the most effective at enhancing permeability.

Microneedles are another way to enhance the permeation of nanoparticles into the skin. The mechanism of transdermal delivery of nanoencapsulated across microneedle-treated skin was studied using the rhodamine B (Rh B) and FITC as model hydrophilic and hydrophobic small/medium-size molecules, 
respectively [115]. Permeation of the model dyes encapsulated in PLGA nanoparticles through porcine skin pretreated with a microneedle array was affected by the physicochemical characteristics of nanoparticles and the encapsulated dyes. Confocal laser scanning microscopy images showed dye-rich reservoirs, suggesting a mechanism involving the influx of nanoparticles deep into microneedle-created channels. The results showed even dye flux was enhanced by nanoparticles with a smaller particle size, hydrophilicity, and a negative zeta potential.

Nanoparticles can interact with the skin at a cellular level, and this interaction can be used to enhance immune reactivity for topical vaccine applications [103]. Considering this statement, dissolving microneedle arrays loaded with nanoencapsulated (PLGA nanoparticles) antigen were evaluated regarding their efficacy in increasing vaccine immunogenicity by targeting the antigen specifically to contiguous dendritic cell networks within the skin [116]. The results showed that the antigen-encapsulated nanoparticles were delivered from skin dendritic cells to cutaneous draining lymph nodes, where they subsequently induced significant antigen-specific $\mathrm{T}$ cell proliferation. Antigen-encapsulated nanoparticle vaccination via microneedles induced antigen-specific cellular immune responses in mice. Furthermore, the activation of antigen-specific cytotoxic CD8+ $\mathrm{T}$ cells induced protection in vivo against both the development of antigen-expressing B16 melanoma tumors and a murine model of para-influenza.

Another proposal to increase the ratio of the drug in the target tissue is based on follicular drug delivery carriers. The hair follicle has been shown to be not only an important penetration route for nanoparticles, but also a significant long-term reservoir. In hair follicles, nanoparticles are surrounded by a dense network of blood capillaries, which is important for drug delivery and systemic uptake. Even differentiated targeting of specific follicular structures can be achieved, since the penetration depth of nanoparticles can be influenced by their size [117]. It is important consider that the movement of the hairs caused by massage push the nanoparticles deeper into the hair follicles. Therefore, massage after the application of nanoparticles may be necessary [102].

In this context, Raber and co-workers [118] quantified the uptake of fluorescently-labeled PLGA nanoparticles into hair follicles using in vitro (pig ear) and in vivo (human volunteers) models. The follicular uptake of the nanoparticles was dependent of the surface modifications (plain PLGA, CS-coated PLGA, or CS-PLGA coated with different phospholipids). Plain PLGA nanoparticles with a negative zeta potential, as well as dipalmitoyl phosphatidylcholine (DPPC) and DPPC:1,2-dioleoyl-3-trimethylammonium-propane (DOTAP) (92:8)-coated CS-PLGA nanoparticles presented follicular uptake to a greater extent than CS-PLGA nanoparticles and DPPC: cholesterol (85: 15)-coated CS-PLGA nanoparticles, which may indicate that a negative surface charge as well as lipophilic surface properties may facilitate follicular uptake. 
The penetration and storage behavior of dye-containing nanoparticles in hair follicles and the dye in the non-particulate form were evaluated in vitro in porcine skin [119]. When massage was applied, the nanoparticles penetrated much deeper into the hair follicles than the dye in the non-particulate form. In addition, a differential stripping assay was carried out in vivo on human skin, showing that the nanoparticles were stored in hair follicles up to 10 days, while the free dye could only be detected for up to 4 days. These results indicate that hair follicles could be used as a reservoir for the topical administration of active molecules.

\subsection{CONCLUSION}

Drug-loaded nanoparticles have emerged as one of the most important applications in medicine. These innovative systems present physical properties that can be exploited to overcome anatomical and physiological barriers associated with drug delivery. When administered by the parenteral route, the pharmacological effects of the nanoencapsulated drug can be improved, the side effects can be reduced, and a specific site in the body can be reached using an active targeting approach. By oral administration, nanoparticles are able to enhance intestinal permeability, control drug delivery, and protect drugs in the gastrointestinal tract, whereas by nasal administration, nanosystems can improve local absorption on several levels. In addition, cutaneous application of nanoparticles can release the compound within the skin or allow for permeation through the skin, acting as a dermal or transdermal carrier system.

Thus, nanoparticles can be used to increase drug bioavailability, to induce drug accumulation at a specific site of the body, and to decrease drug side effects, leading to improved therapeutic effectiveness and increased patient adherence to treatment. 


\section{REFERENCES}

1. A.C. Hunter, J. Elsom, P.P. Wibroe, S.M. Moghimi. Maturitas 73 (2012) 5-18.

2. A. Bernkop-Schnurch. Eur. J. Pharm. Sci. 49 (2013) 272-277.

3. E.M. Merisko-Liversidge, G.G. Liversidge. Toxicol. Pathol. 36 (2008) 43-48.

4. I. Limayem Blouza, C. Charcosset, S. Sfar, H. Fessi. Int. J. Pharm. 325 (2006) 124-131.

5. D.A. Chiappetta, G. Facorro, E.R. de Celis, A. Sosnik. Nanomed. Nanotechnol. 7 (2011) 624-637.

6. C.F. Giordani, D. de Souza, L. Dornelles, C.W. Nogueira, M.P. Alves, M. Prigol, O.E. Rodrigues. Appl. Biochem. Biotechnol. 172 (2014) 755-766.

7. L. Martin-Banderas, J. Alvarez-Fuentes, M. Duran-Lobato, J. Prados, C. Melguizo, M. Fernandez-Arevalo, M.A. Holgado. Int. J. Nanomedicine 7 (2012) 5793-5806.

8. M. Morgen, C. Bloom, R. Beyerinck, A. Bello, W. Song, K. Wilkinson, R. Steenwyk, S. Shamblin. Pharm. Res. 29 (2012) 427-440.

9. U. Shah, G. Joshi, K. Sawant. Mater. Sci. Eng. C Mater. Biol. Appl. 35 (2014) 153-163.

10. K. Bowman, K.W. Leong. Int. J. Nanomedicine 1 (2006) 117-128.

11. M.C. Chen, F.L. Mi, Z.X. Liao, C.W. Hsiao, K. Sonaje, M.F. Chung, L.W. Hsu, H.W. Sung. Adv. Drug Deliv. Rev. 65 (2013) 865-879.

12. S. Saremi, R. Dinarvand, A. Kebriaeezadeh, S.N. Ostad, F. Atyabi. Biomed. Res. Int. 2013 (2013) 150478.

13. M. Guo, W.T. Rong, J. Hou, D.F. Wang, Y. Lu, Y. Wang, S.Q. Yu, Q. Xu. Nanotechnology 24 (2013) 245101.

14. M.R. Rekha, C.P. Sharma. J. Control. Release 135 (2009) 144-151.

15. X. Xie, Q. Tao, Y. Zou, F. Zhang, M. Guo, Y. Wang, H. Wang, Q. Zhou, S. Yu. J. Agric. Food Chem. 59 (2011) 9280-9289.

16. G. Joshi, A. Kumar, K. Sawant. Eur. J. Pharm. Sci. 60 (2014) 80-89.

17. P. Zakeri-Milani, B.D. Loveymi, M. Jelvehgari, H. Valizadeh. Colloids Surf. B 103 (2013) 174-181.

18. E. Roger, S. Kalscheuer, A. Kirtane, B.R. Guru, A.E. Grill, J. Whittum-Hudson, J. Panyam. Mol. Pharm. 9 (2012) 2103-2110.

19. K. Sonaje, J.L. Italia, G. Sharma, V. Bhardwaj, K. Tikoo, M.N. Kumar. Pharm. Res. 24 (2007) 899-908.

20. A. Dube, J.A. Nicolazzo, I. Larson. Eur. J. Pharm. Sci. 44 (2011) 422-426.

21. A. Makhlof, Y. Tozuka, H. Takeuchi. Eur. J. Pharm. Sci. 42 (2011) 445-451.

22. J. Dou, H. Zhang, X. Liu, M. Zhang, G. Zhai. Colloids Surf. B 114 (2014) 20-27.

23. R. Diab, C. Jaafar-Maalej, H. Fessi, P. Maincent. AAPS J. 14 (2012) 688-702.

24. S.K. Yadav, S. Mishra, B. Mishra. AAPS PharmSciTech 13 (2012) 1031-1044.

25. Z.M. Wu, L. Zhou, X.D. Guo, W. Jiang, L. Ling, Y. Qian, K.Q. Luo, L.J. Zhang. Int. J. Pharm. 425 (2012) 1-8.

26. M. Sharma, V. Sharma, A.K. Panda, D.K. Majumdar. Int. J. Nanomedicine 6 (2011) 2097-2111.

27. L. Illum. J. Pharm. Sci. 96 (2007) 473-483.

28. A. Pires, A. Fortuna, G. Alves, A. Falcão. J. Pharm. Pharm. Sci. 12 (2009) 288-311.

29. P.G. Djupesland, J.C. Messina, R.A. Mahmoud. Ther. Deliv. 5 (2014) 709-733.

30. L. Casettari, L. Illum. J. Control. Release 190 (2014) 189-200. 
31. F.M. Goycoolea, G. Lollo, C. Remunan-Lopez, F. Quaglia, M.J. Alonso. Biomacromolecules 10 (2009) 1736-1743.

32. X. Wang, N. Chi, X. Tang. Eur. J. Pharm. Biopharm. 70 (2008) 735-740.

33. X. Zhang, H. Zhang, Z. Wu, Z. Wang, H. Niu, C. Li. Eur. J. Pharm. Biopharm. 68 (2008) 526-534.

34. D. Teijeiro-Osorio, C. Remunan-Lopez, M.J. Alonso. Biomacromolecules 10 (2009) 243-249.

35. C.B. Woitiski, B. Sarmento, R.A. Carvalho, R.J. Neufeld, F. Veiga. Int. J. Pharm. 412 (2011) 123-131.

36. A.H. Krauland, V.M. Leitner, V. Grabovac, A. Bernkop-Schnurch. J. Pharm. Sci. 95 (2006) 2463-2472.

37. A. Jintapattanakit, P. Peungvicha, A. Sailasuta, T. Kissel, V.B. Junyaprasert. J. Pharm. Pharmacol. 62 (2010) 583-591.

38. X. Zhang, Y. Wang, C. Zheng, C. Li. Eur. J. Pharm. Biopharm. 82 (2012) 76-84.

39. X. Wang, C. Zheng, Z. Wu, D. Teng, X. Zhang, Z. Wang, C. Li. J. Biomed. Mater. Res. B Appl. Biomater. 88 (2009) 150-161.

40. N. Csaba, M. Garcia-Fuentes, M.J. Alonso. Adv. Drug Deliv. Rev. 61 (2009) 140-157.

41. W.M. Pardridge. NeuroRx 2 (2005) 3-14.

42. R. Daneman, A. Prat. Cold Spring Harb. Perspect. Biol. 7(1) (2015) a020412.

43. A. Mistry, S. Stolnik, L. Illum. Int. J. Pharm. 379 (2009) 146-157.

44. L. Kozlovskaya, M. Abou-Kaoud, D. Stepensky. J. Control. Release 189 (2014) 133-140.

45. S. Alam, Z.I. Khan, G. Mustafa, M. Kumar, F. Islam, A. Bhatnagar, F.J. Ahmad. Int. J. Nanomedicine 7 (2012) 5705-5718.

46. M. Fazil, S. Md, S. Haque, M. Kumar, S. Baboota, J.K. Sahni, J. Ali. Eur. J. Pharm. Sci. 47 (2012) 6-15.

47. M. Kumar, R.S. Pandey, K.C. Patra, S.K. Jain, M.L. Soni, J.S. Dangi, J. Madan. Int. J. Biol. Macromol. 61 (2013) 189-195.

48. X. Gao, W. Tao, W. Lu, Q. Zhang, Y. Zhang, X. Jiang, S. Fu. Biomaterials 27 (2006) 3482-3490.

49. X. Gao, J. Chen, W. Tao, J. Zhu, Q. Zhang, H. Chen, X. Jiang. Int. J. Pharm. 340 (2007) 207-215.

50. Q. Liu, X. Shao, J. Chen, Y. Shen, C. Feng, X. Gao, Y. Zhao, J. Li, Q. Zhang, X. Jiang. Toxicol. Appl. Pharmacol. 251 (2011) 79-84.

51. Q. Liu, Y. Shen, J. Chen, X. Gao, C. Feng, L. Wang, Q. Zhang, X. Jiang. Pharm. Res. 29 (2012) 546-558.

52. Z. Liu, M. Jiang, T. Kang, D. Miao, G. Gu, Q. Song, L. Yao, Q. Hu, Y. Tu, Z. Pang, H. Chen, X. Jiang, X. Gao, J. Chen. Biomaterials 34 (2013) 3870-3881.

53. T. Kanazawa, F. Akiyama, S. Kakizaki, Y. Takashima, Y. Seta. Biomaterials 34 (2013) 9220-9226.

54. Y. Shen, J. Chen, Q. Liu, C. Feng, X. Gao, L. Wang, Q. Zhang, X. Jiang. Int. J. Pharm. 413 (2011) 184-193.

55. L. Chen, J. Zhu, Y. Li, J. Lu, L. Gao, H. Xu, M. Fan, X. Yang. PLoS One 8 (2013) e71953.

56. G. Feng, Q. Jiang, M. Xia, Y. Lu, W. Qiu, D. Zhao, L. Lu, G. Peng, Y. Wang. PLoS One 8 (2013) e61135.

57. D. Raghuwanshi, V. Mishra, D. Das, K. Kaur, M.R. Suresh. Mol. Pharm. 9 (2012) 946-956. 
58. J. Xu, W. Dai, Z. Wang, B. Chen, Z. Li, X. Fan. Clin. Vaccine Immunol. 18 (2011) 75-81.

59. Z. Liu, H. Guo, Y. Wu, H. Yu, H. Yang, J. Li. Int. Arch. Allergy Immunol. 150 (2009) 221-228.

60. W. Yao, Y. Peng, M. Du, J. Luo, L. Zong. Mol. Pharm. 10 (2013) 2904-2914.

61. N.K. Gupta, P. Tomar, V. Sharma, V.K. Dixit. Vaccine 29 (2011) 9026-9037.

62. M. Tafaghodi, V. Saluja, G.F. Kersten, H. Kraan, B. Slutter, J.P. Amorij, W. Jiskoot. Vaccine 30 (2012) 5341-5348.

63. C. Keijzer, B. Slutter, R. van der Zee, W. Jiskoot, W. van Eden, F. Broere. PLoS One 6 (2011) e26684.

64. R.J. Verheul, B. Slutter, S.M. Bal, J.A. Bouwstra, W. Jiskoot, W.E. Hennink. J. Control. Release 156 (2011) 46-52.

65. S. Mangal, D. Pawar, N.K. Garg, A.K. Jain, S.P. Vyas, D.S. Rao, K.S. Jaganathan. Vaccine 29 (2011) 4953-4962.

66. B. Slutter, W. Jiskoot. J. Control. Release 148 (2010) 117-121.

67. B. Slutter, S.M. Bal, I. Que, E. Kaijzel, C. Lowik, J. Bouwstra, W. Jiskoot. Mol. Pharm. 7 (2010) 2207-2215.

68. B. Sayin, S. Somavarapu, X.W. Li, D. Sesardic, S. Senel, O.H. Alpar. Eur. J. Pharm. Sci. 38 (2009) 362-369.

69. D. Pawar, S. Mangal, R. Goswami, K.S. Jaganathan. Eur. J. Pharm. Biopharm. 85 (2013) 550-559.

70. A.I. Camacho, J.M. Irache, J. de Souza, S. Sanchez-Gomez, C. Gamazo. Vaccine 31 (2013) 3288-3294.

71. R. Da Costa Martins, C. Gamazo, M. Sanchez-Martinez, M. Barberan, I. Penuelas, J.M. Irache. J. Control. Release 162 (2012) 553-560.

72. R. Da Costa Martins, C. Gamazo, J.M. Irache. Eur. J. Pharm. Sci. 37 (2009) 563-572.

73. E. Merisko-Liversidge, G.G. Liversidge. Adv. Drug Deliv. Rev. 63 (2011) 427-440.

74. M. Rowland. J. Pharm. Sci. 61 (1972) 70-74.

75. L. Zhang, N. Zhang. Int. J. Nanomed. 8 (2013) 2927-2941.

76. J. Kreuter. Adv. Drug Deliv. Rev. 71 (2014) 2-14.

77. P. Legrand, G. Barratt, V. Mosqueira, H. Fessi, J.P. Devissaguet. Stp Pharma. Sci. 9 (1999) 411-418.

78. D. Torrecilla, M.V. Lozano, E. Lallana, J.I. Neissa, R. Novoa-Carballal, A. Vidal, E. Fernandez-Megia, D. Torres, R. Riguera, M.J. Alonso, F. Dominguez. Eur. J. Pharm. Biopharm. 83 (2013) 330-337.

79. S. Ballot, N. Noiret, F. Hindre, B. Denizot, E. Garin, H. Rajerison, J.P. Benoit. Eur. J. Nucl. Med. Mol. Imaging 33 (2006) 602-607.

80. F. Danhier, E. Ansorena, J.M. Silva, R. Coco, A. Le Breton, V. Preat. J. Control. Release 161 (2012) 505-522.

81. J. Agnihotri, N.K. Jain. Artif. Cell Nanomed. Biotechnol. 41 (2013) 309-314.

82. E.A. Bender, M.D. Adorne, L.M. Colome, D.S.P. Abdalla, S.S. Guterres, A.R. Pohlmann. Int. J. Pharm. 426 (2012) 271-279.

83. V.C.F. Mosqueira, P. Legrand, J.L. Morgat, M. Vert, E. Mysiakine, R. Gref, J.P. Devissaguet, G. Barratt. Pharm. Res.-Dordr. 18 (2001) 1411-1419.

84. T.M. Allen. Adv. Drug Deliv. Rev. 13 (1994) 285-309.

85. P. Couvreur, C. Vauthier. Pharm. Res.-Dordr. 23 (2006) 1417-1450. 
86. F.M. Veronese, R. Mendichi, O. Schiavon, G. Pasut, L. Andersson, A. Tsirk, J. Ford, G. Wu, S. Kneller, J. Davies, R. Duncan. Bioconjugate Chemistry 16 (2005) 775-784.

87. S. Parveen, S.K. Sahoo. Eur. J. Pharmacol. 670 (2011) 372-383.

88. M. Gagliardi. J. Appl. Polym. Sci. 132(3) (2015) 41310.

89. K.J. Cho, X. Wang, S.M. Nie, Z. Chen, D.M. Shin. Clin. Cancer Res. 14 (2008) 1310-1316.

90. P. Couvreur, C. Dubernet, F. Puisieux, J. Robert. Pathol. Biol. 42 (1994) 923-923.

91. Q. Liu, R.R. Li, Z.S. Zhu, X.P. Qian, W.X. Guan, L.X. Yu, M. Yang, X.Q. Jiang, B.R. Liu. Int. J. Pharm. 430 (2012) 350-358.

92. V.C.F. Mosqueira, P.M. Loiseau, C. Bories, P. Legrand, J.P. Devissaguet, G. Barratt. Antimicrob. Agents Chemother. 48 (2004) 1222-1228.

93. J.L. Arias, J.D. Unciti-Broceta, J. Maceira, T. del Castillo, J. Hernandez-Quero, S. Magez, M. Soriano, J.A. Garcia-Salcedo. J. Control. Release 197 (2015) 190-198.

94. E.A. Bender, M.F. Cavalcante, M.D. Adorne, L.M. Colome, S.S. Guterres, D.S.P. Abdalla, A.R. Pohlmann. Pharm. Res.-Dordr. 31 (2014) 2975-2987.

95. A. Llevot, D. Astruc. Chem. Soc. Rev. 41 (2012) 242-257.

96. M. Sarparanta, L.M. Bimbo, J. Rytkönen, E. Mäkilä, T.J. Laaksonen, P. Laaksonen, M. Nyman, J. Salonen, M.B. Linder, J. Hirvonen, H.A. Santos, A.J. Airaksinen. Mol. Pharmaceut. 9 (2012) 654-663.

97. S.N. Tammam, H.M.E. Azzazy, A. Lamprecht. J. Biomed. Nanotechnol. 11 (2015) 555-577.

98. J.M. Raquez, Y. Habibi, M. Murariu, P. Dubois. Prog. Polym. Sci. 38 (2013) 1504-1542.

99. T.K. Yeung, J.W. Hopewell, R.H. Simmonds, L.W. Seymour, R. Duncan, O. Bellini, M. Grandi, F. Spreafico, J. Strohalm, K. Ulbrich. Cancer Chemother. Pharm. 29 (1991) 105-111.

100. D. Schrama, R.A. Reisfeld, J.C. Becker. Nat. Rev. Drug Discov. 5 (2006) 147-159.

101. K.R. Pawar, R.J. Babu. Crit. Rev. Ther. Drug 27 (2010) 419-459.

102. R.H.H. Neubert. Eur. J. Pharm. Biopharm. 77 (2011) 1-2.

103. T.W. Prow, J.E. Grice, L.L. Lin, R. Faye, M. Butler, W. Becker, E.M.T. Wurm, C. Yoong, T.A. Robertson, H.P. Soyer, M.S. Roberts. Adv. Drug Deliv. Rev. 63 (2011) 470-491.

104. K. Tomoda, N. Yabuki, H. Terada, K. Makino. Colloid Polym. Sci. 292 (2014) 3195-3203.

105. S. Arayachukeat, S.P. Wanichwecharungruang, T. Tree-Udom. Int. J. Pharm. 404 (2011) 281-288.

106. M. Marimuthu, D. Bennet, S. Kim. Polym. J. 45 (2013) 202-209.

107. V. Vijayan, K.R. Reddy, S. Sakthivel, C. Swetha. Colloid Surf. B 111 (2013) 150-155.

108. R.V. Contri, T. Katzer, A.F. Ourique, A.L.M. da Silva, R.C.R. Beck, A.R. Pohlmann, S.S. Guterres. J. Biomed. Nanotechnol. 10 (2014) 820-830.

109. I. Ozcan, E. Azizoglu, T. Senyigit, M. Ozyazici, O. Ozer. J. Drug Target. 21 (2013) 542-550.

110. M.L. Gou, L. Wu, Q.Q. Yin, Q.F. Guo, G. Guo, J. Liu, X. Zhao, Y.Q. Wei, Z.Y. Qian. J. Nanosci. Nanotechnol. 9 (2009) 6360-6365. 
111. M. Morgen, G.W. Lu, D. Du, R. Stehle, F. Lembke, J. Cervantes, S. Ciotti, R. Haskell, D. Smithey, K. Haley, C. Fan. Int. J. Pharm. 416 (2011) 314-322.

112. Z. Hussain, H. Katas, M.C.I.M. Amin, E. Kumulosasi, S. Sahudin. J. Pharm. Sci. 102 (2013) 1063-1075.

113. P.W. Lee, S.H. Hsu, J.S. Tsai, F.R. Chen, P.J. Huang, C.J. Ke, Z.X. Liao, C.W. Hsiao, H.J. Lin, H.W. Sung. Biomaterials 31 (2010) 2425-2434.

114. Z. Zhang, P.C. Tsai, T. Ramezanli, B.B. Michniak-Kohn, Wires Nanomed. Nanobi. 5 (2013) 205-218.

115. Y.A. Gomaa, M.J. Garland, F.J. McInnes, R.F. Donnelly, L.K. El-Khordagui, C.G. Wilson. Eur. J. Pharm. Biopharm. 86 (2014) 145-155.

116. M. Zaric, O. Lyubomska, O. Touzelet, C. Poux, S. Al-Zahrani, F. Fay, L. Wallace, D. Terhorst, B. Malissen, S. Henri, U.F. Power, C.J. Scott, R.F. Donnelly, A. Kissenpfennig. Acs Nano 7 (2013) 2042-2055.

117. J. Lademann, H. Richter, S. Schanzer, F. Knorr, M. Meinke, W. Sterry, A. Patzelt. Eur. J. Pharm. Biopharm. 77 (2011) 465-468.

118. A.S. Raber, A. Mittal, J. Schafer, U. Bakowsky, J. Reichrath, T. Vogt, U.F. Schaefer, S. Hansen, C.M. Lehr. J. Control. Release 179 (2014) 25-32.

119. J. Lademann, H. Richter, A. Teichmann, N. Otberg, U. Blume-Peytavi, J. Luengo, B. Weiss, U.F. Schaefer, C.M. Lehr, R. Wepf, W. Sterry. Eur. J. Pharm. Biopharm. 66 (2007) 159-164. 
Chapter 2 